\title{
Critical Limb Ischaemia for Thrombosed Common Femoral Artery Aneurysm: A Successful Hybrid Treatment
}

\author{
Orellana Dávila Bernardo, MD*, Fresilli Mauro, MD, Oddi Fabio Massimo, MD, Diotallevi \\ Nicolò, MD, Ascoli Marchetti Andrea and Ippoliti Arnaldo
}

Department of Surgery, Division of Vascular Surgery, Policlinico Tor Vergata, Rome, Italy

*Corresponding author: Orellana Dávila Bernardo, MD, Department of Surgery, Division of Vascular Surgery, Viale Oxford 81, Policlinico Tor Vergata, 00133 Rome, Italy, Tel: +393427247215

\begin{abstract}
True atherosclerotic aneurysms of the common femoral artery (CFA) are rare and often associated with other peripheral or aortic aneurysms. Degenerative aneurysms of the lower extremity most commonly involve the popliteal artery, while they are rarely detected in the femoral region. In this region, aneurysms most frequently involve the common femoral artery (CFA), whereas true aneurysms of the superficial femoral artery (SFA) represent only $15 \%$ to $25 \%$ of femoral arterial aneurysms.

We report a case of an 84-year-old patient presenting a degenerative thrombosed aneurysm of the CFA and critical limb ischemia. After an accurate workout, the patient underwent successful aneurysmectomy and bypass grafting, with a satisfying mild-term follow-up and patency of the graft. The patient came back presenting surgical site infection (SSI) and wound dehiscence that was successfully treated with the Vacuum Assisted Therapy (V.A.C. ${ }^{\circledR}$ therapy) and accurate medical therapy.

Aneurysmectomy and revascularization of the distal CFA with an artificial blood vessel graft were performed, associated with revascularization and stenting of the left femoral-iliac axis. CFA aneurysms are rare and have a low rate of rupture but high risk of complications like embolic or thrombotic lower limb ischemia with challenging treatment solutions. Nowadays open vascular surgery, sometimes in association with the endovascular procedure, plays an important role in the treatment of this pathology.
\end{abstract}

\section{Abbreviations}

CFA: Common Femoral Artery; SFA: Superficial Femoral Artery; EIA: External Iliac Artery; PFA: Profunda Femoris Artery; VAC: Vacuum Assisted Closure; COPD: Chronic Obstructive Pulmonary Disease; CLTI Critical-Limbs Threatening Ischemia; FAA: True femoral artery aneurysms; PTFE: Polytetrafluoroethylene; NPWT: Negative Pressure Wound Therapy; SSI: Surgical Site Infection

\section{Introduction}

True atherosclerotic aneurysms of the common femoral artery (CFA) are rare and often associated with other peripheral or aortic aneurysms. Degenerative aneurysms of the lower extremity most commonly involve the popliteal artery, while they are rarely detected in the femoral region thus, there are a little number of cases reported in the literature and its natural history is not well defined [1-3]. As with any peripheral or a visceral aneurysm, the main risks associated with femoral artery aneurysms include thrombosis/ occlusion, fistulization, distal embolization, and rupture. We present a case of thrombosed common femoral artery aneurysm, treated with a hybrid technique [1-3].

\section{Case Report}

An 84-year-old smoker man presented claiming for pain to the left leg appearing at rest. Physical examination revealed no left femoral and peripheral pulses, a necrotic ulcer compromising all the $1^{\text {st }}$ left toe and a mass was discovered to the left groin. A Duplex scan revealed a thrombosed aneurysm of the left CFA and demodulated flow on the distal vessels, till the tibial arteries. Computed tomography showed a small aortic abdominal aneurysm (DTM $35 \mathrm{~mm}$ ), complete ipsilateral external iliac artery (EIA) occlusion, thrombosed CFA aneurysm of $27 \mathrm{~mm}$ in diameter (Figure 1). The profunda femoris artery (PFA) was patent with ostial stenosis and poor collateral vessels that held the SFA and distal arteries perfusion. The patient had no history of trauma, previous operations,

Citation: Bernardo OD, Mauro F, Massimo OF, Nicolò D, Andrea AM, et al. (2021) Critical Limb Ischaemia for Thrombosed Common Femoral Artery Aneurysm: A Successful Hybrid Treatment. Int Arch Vasc Med 4:012. doi.org/10.23937/2690-3164/1710012

Accepted: December 11, 2021: Published: December 13, 2021

Copyright: (c) 2021 Bernardo OD, et al. This is an open-access article distributed under the terms of the Creative Commons Attribution License, which permits unrestricted use, distribution, and reproduction in any medium, provided the original author and source are credited. 

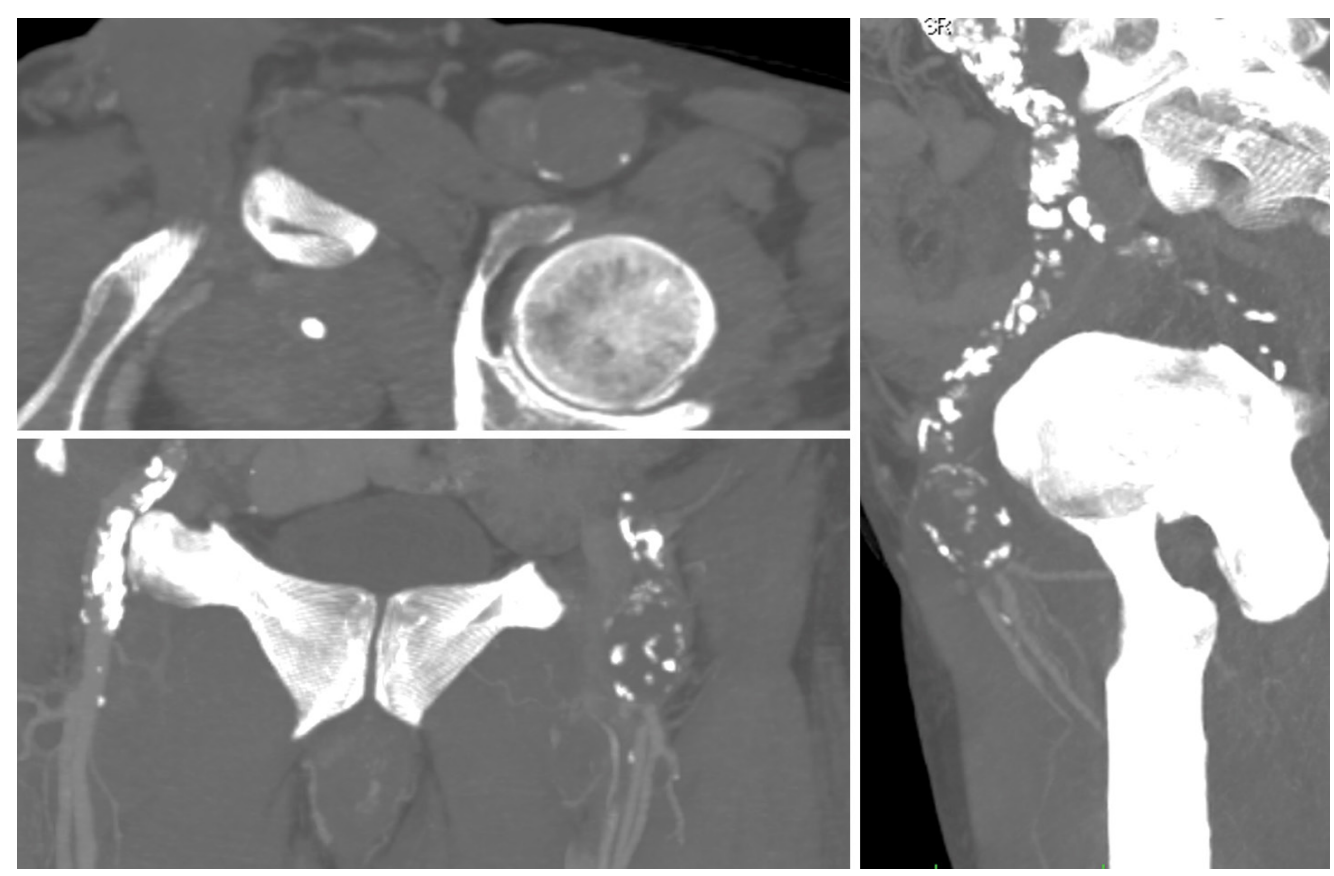

Figure 1: Pre-operative CTA Multi-Planar-Reconstruction (MPR) showing thrombosed CFA aneurysm, EIA occlusion, patent of PFA and SFA.
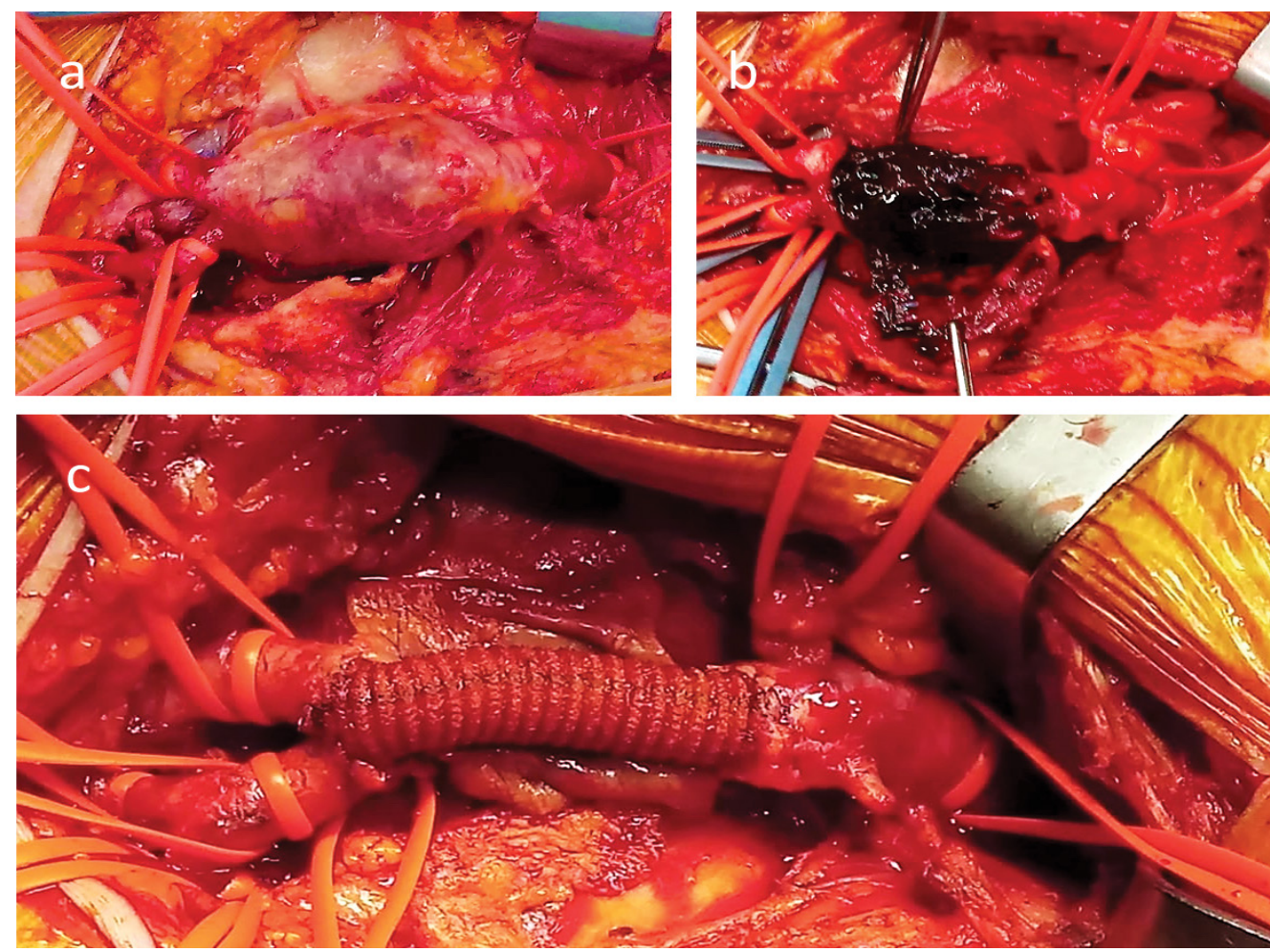

Figure 2: (A) Aneurysmatic CFA until its bifurcation; (B) Opened aneurysmal sac; (C) T-T anastomosis with a Dacron Silver $10 \mathrm{~mm}$ graft from the CFA origin to the origin of the bifurcation.

or interventions. He had medication-controlled hypertension and untreated COPD. The ankle/brachial index was 0.3 on the posterior tibial artery and wasn't measurable on the anterior tibial artery. All these findings lay for critical-limb threatening ischemia (CLTI) due to the CFA aneurysm thrombosis, with retrograde EIA occlusion, and the surgical treatment was deemed mandatory to avoid a major lower limb amputation. Due to age and comorbidities, with a prohibiting surgical risk, the iliac-femoral bypass surgery was excluded, and a hybrid procedure was planned.

Under local anesthesia, a longitudinal groin incision was performed showing no pulsatile CFA aneurysm of 25 $\mathrm{mm}$ approximately. The proximal neck of the aneurysm was isolated for about $2 \mathrm{~cm}$ distal to the inguinal ligament. The aneurysm involved the SFA and PFA origins that were exposed and surrounded with vessel loops, as well 
as some large collateral vessels that were recognized and preserved (Figure 2A). After clamping the PFA and SFA distal to the aneurysm, we opened the sac and removed a massive quantity of atherosclerotic thrombus (Figure 2B). After eversion endarterectomy of the PFA ostium, acceptable blood reflux was detected coming from both the SFA and PFA. An over-the-wire radio-guided Fogarty embolectomy of the left iliac axis was performed to restore an adequate in-flow, attempting to preserve the left hypogastric artery patency. Femoral-to-femoral with a Silver Dacron $8 \mathrm{~mm}$ graft replacement was performed in a standard fashion (Figure $2 \mathrm{C}$ ). Angiography with a direct puncture of the bypass showed the persistence of thrombus apposition, angioplasty with a Gore Viabahn $9 \times 10 \mathrm{~mm}$ stenting of the whole EIA and an adjunctive ev3 Protègè $\mathrm{GPS}^{\circ} 12 \times 60 \mathrm{~mm}$ in the common iliac artery to preserve the internal iliac artery was performed.
The final angiography showed patency of both stents, of the femoral-to-femoral bypass, and of the femoral bifurcation, in absence of residual stenosis to the iliac-femoral axis (Figure 3). The postoperative clinical examination revealed the presence of the popliteal and peripheral pulses with an arterial/brachial index was 0.8 on the posterior tibial artery and 0.6 on the anterior tibial artery.

Following surgery, the patient was discharged in good clinical conditions. The patient didn't show up to the control and wound care that was programmed. Two weeks later the patient presents to our service with important lymphorrhagia and wound dehiscence. The wound culture was performed, showing a staphylococcus infection. After two weeks under V.A.C. ${ }^{\circ}$ therapy and targeted antibiotics, the culture test results negative and the inguinal wound has healed.
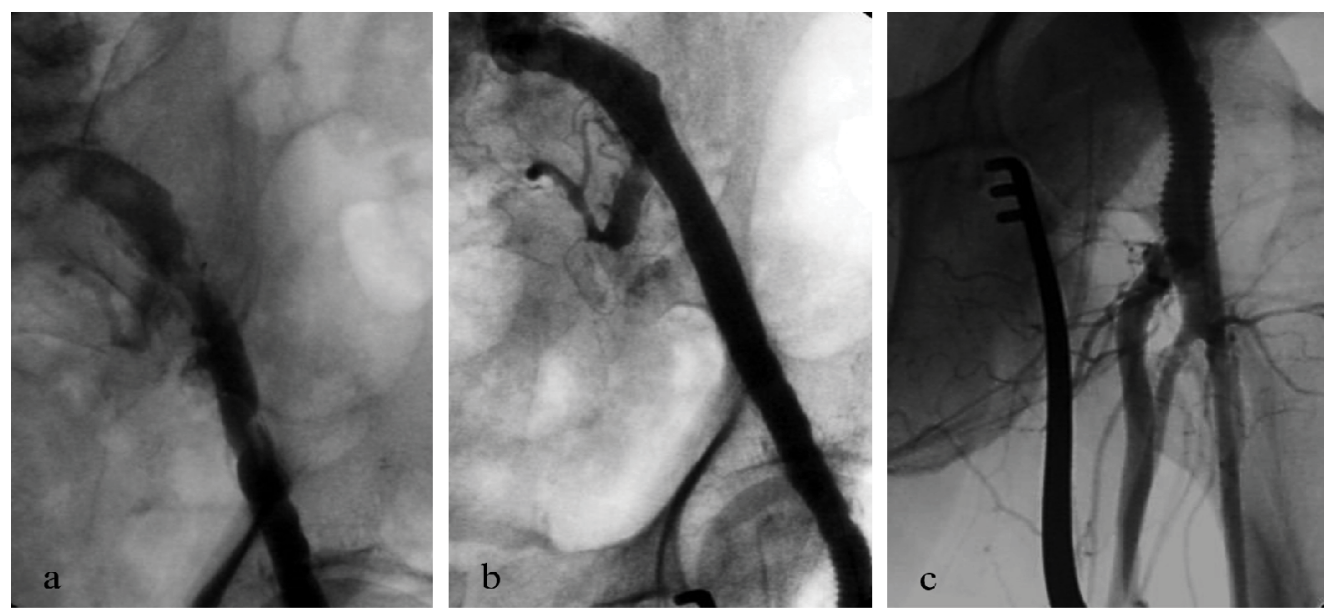

Figure 3: Intraoperative angiography showing the patency of EIA, bypass, SFA and PFA.
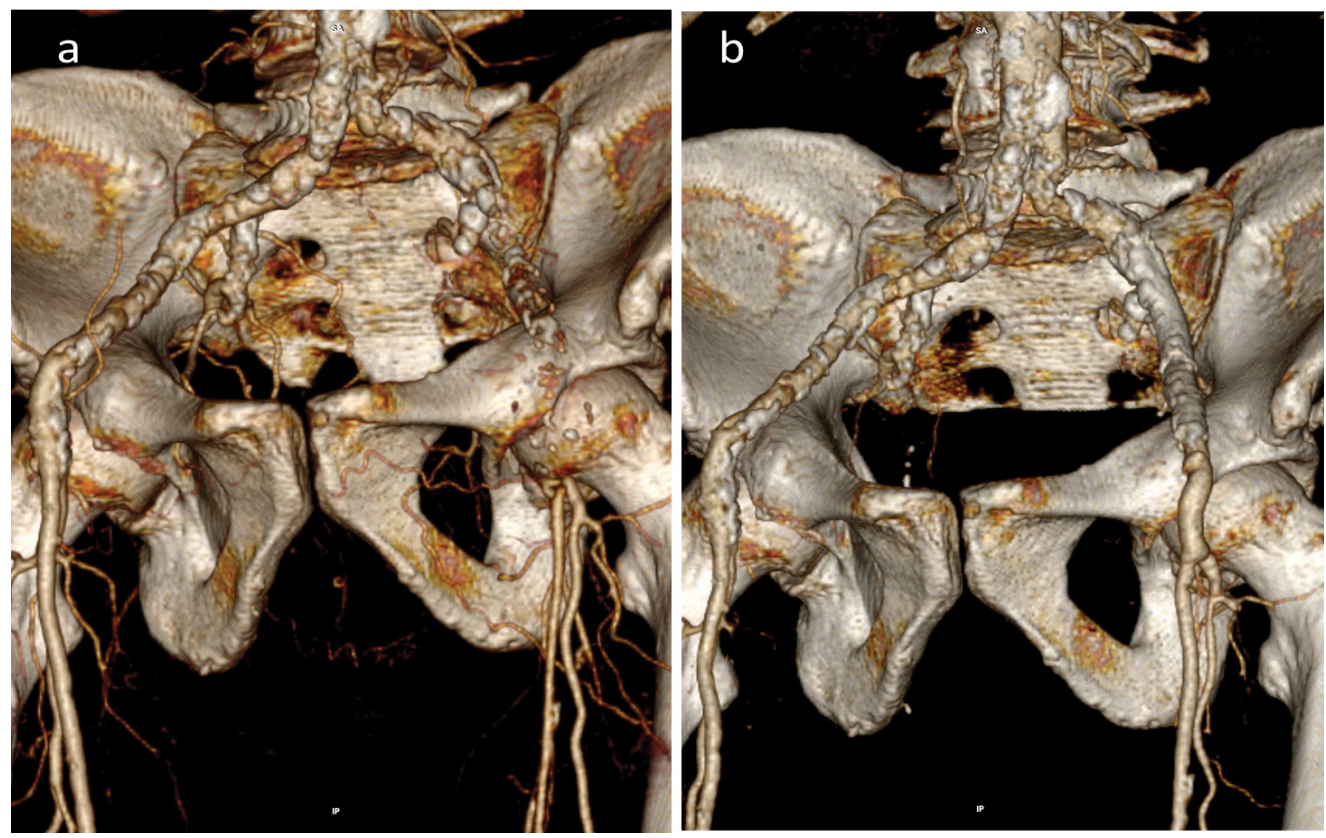

Figure 4: (A) CTA showed an aortic abdominal aneurysm (DTM $35 \mathrm{~mm}$ ), complete ipsilateral EIA occlusion, patent PFA with ostium stenosis and poor collateral vessels that held the SFA and distal arteries perfusion; (B) Follow-up CTA scan showed good blood flow in the graft, EIA and SFA. 
A follow-up multi detector computed tomography scan and 3D reconstruction showed the pre and the post surgery condition of the patient (Figure 4). At the last control after 6 months of follow-up, the patient has a wound completely healed without signs of necrosis progression at the left toe.

\section{Discussion}

The Society of Vascular Surgery defines a true aneurysm as a focal, isolated arterial dilation that includes all three layers of the vessel wall and measures at least 1.5 times the diameter of the disease-free proximal, adjacent segment. [4,5].

True femoral artery aneurysms (FAA) are less common than pseudoaneurysms and are often associated with popliteal artery and aortic aneurysms. It can be caused by the weakening of the arterial wall, most often secondary to atherosclerosis. Traumatic aneurysmal change can occur either through direct trauma, iatrogenesis (angioplasty, groin surgery), or intravenous drug abuse [1-3].

The most complex femoral aneurysms are infected pseudoaneurysms most of the result as a complication of percutaneous femoral access or disruption of surgical bypass anastomoses. These lesions can be challenging to repair and are often not amenable to endovascular techniques.

FAAs have been classified based on anatomic site and relationship to the femoral bifurcation in [6]: Type $I$ as one involving only the FA and terminating before the bifurcation, and type II involving the origin of the profunda femoris and beyond. Another classification system includes isolated deep femoral and superficial femoral aneurysms as well, type III and type IV, respectively [7].

Most FAAs are asymptomatic at presentation, but patients can develop a variety of symptoms related to expansion or thrombosis. FAAs can also present with limb-threatening complications due to occlusion, distal embolization, or rupture, although the incidence of acute limb ischemia is lower for femoral compared with popliteal artery aneurysms. FAA is more commonly seen in individuals who are older than 70 years of age and male $[8,9]$. These aneurysms can be bilateral in up to $70 \%$ of cases and up to $25 \%$ of patients can have an abdominal aortic or iliac artery aneurysm. Risk factors for femoral artery aneurysms include smoking, arteriosclerosis, high blood pressure, and systemic connective tissue disorders $[8,10]$.

The decision to repair an FAA primarily depends on the clinical presentation, the aneurysm diameter, and how fit the patient is for open surgery, the predominant method of repair. Consideration should be given to repair of asymptomatic femoral artery aneurysms when they exceed about $2.5 \mathrm{~cm}$ in diameter $[1,11]$. For aneurysms involving the common femoral artery only, the repair is usually performed with an end-to-end interposition graft of Polytetrafluoroethylene (PTFE) or Dacron. For more complicated FAAs of the common femoral artery, the uninvolved branch is implanted into the interposition graft with an end-to-side anastomosis [6]. For aneurysms that involve the origins of both the superficial femoral and profunda femoris arteries, the use of a Dacron bifurcated graft has been described [1].

The endovascular treatment experience is limited. Stent placement in the common femoral artery is generally avoided, given the risk of future stent fracture or migration due to repeated hip flexion [12]. In addition, the short length of the common femoral artery makes it difficult to obtain an adequate seal while preserving flow into both the superficial and deep femoral arteries [13]. Endovascular repair has primarily been performed in urgent situations involving aneurysm rupture, or for patients who are hemodynamically unstable, critically ill, or not likely to tolerate traditional open repair [14].

Covered stents have been used successfully for FAAs of the mid-to-distal superficial femoral artery, a location for which there is generally an adequate zone for sealing, and protection from bending stresses [15]. However, only 10 cases of such repair are reported in the literature, and long-term data are unavailable [16].

Surgical site infection (SSI) and vascular graft infections remain a serious concern in vascular surgery because of large subcutaneous vessel involvement $[17,18]$. SSI occurs in $5-10 \%$ of patients who undergo vascular surgery $[9,19]$ and the incidence of vascular graft infection varies from 1 to $6 \%[20,21]$. Infrainguinal open bypass procedures have the highest infection rates, especially when synthetic prosthetic material is used (10-30\%). The reasons include the groin being a major reservoir of bacteria, infected lymph glands, surgical division of lymphatic channels, the proximity of the groin to the perineum, and the superficial location of vascular grafts in the groin.

The incidence of infrainguinal arterial prosthetic graft infection is $2.5 \%$ [22]. Deep postoperative wound infection with involved vascular grafts increases mortality risk (6-75\%) and limb loss (22-75\%) [23]. Postsurgical infections in vascular surgery are multifactorial, resulting from a complex interplay of patient, surgical, and environmental factors.

Current treatment for vascular complications includes wound observation and debridement as well as topical antibiotics, flaps, and negative pressure wound therapy (NPWT) [23].

The V.A.C. ${ }^{\circ}$ system is a non-invasive method of promoting healing in difficult wounds that fail to respond to other treatment modalities. It is based on the application of negative pressure $(50-150 \mathrm{mmHg})$ by controlled suction to the wound surface, eliminating 
interstitial fluid, which may shorten diffusion distances [23]. Blood perfusion and oxygenation are crucial to ensure proper healing; this system increases microcirculation and capillary blood flow at the wound edge as well as decreasing interstitial edema and stimulating granulation to rapidly fill open wound (reduction of wound size) by diminishing external pressure on capillaries and promote tissue perfusion $[24,25]$. The V.A.C. ${ }^{\circ}$ system reduces bacterial colonization and protects the vascular graft by maintaining its integrity and also has shown to modulate the wound surface and help alleviate patient discomfort $[24,26]$.

\section{Conclusions}

Early diagnosis and management of CFA aneurysms are recommended because of the lower morbidity and mortality rates associated with elective surgery by comparison with emergency procedures. In the majority of the cases, these aneurysms are diagnosed following the onset of complications [27,28], with a high major amputation risk. As in our case, FAAs are most commonly found in elderly, male smokers [29,30], often associated with aneurysms in other districts. A hybrid procedure, combining endovascular and open techniques, limits surgery invasiveness, giving a shorter operating time, and reducing complication rates [31]. Whenever there is a surgical site complication, despite the graft presence, in this case, the V.A.C. ${ }^{\circ}$ therapy has been a safe and effective adjunctive treatment and a very useful component in managing groin dehiscence and wound infections.

\section{Funding}

This research did not receive any specific grant from funding agencies in the public, commercial, or not-forprofit sectors.

\section{Conflict of Interest}

The authors declare that they have no potential conflict of interest in the subject matter or materials discussed in this manuscript.

\section{Informed Consent}

Informed consent has been obtained directly from the patient, for publication of the case report and accompanying images.

\section{Author's Contribution}

$\mathrm{OB}$ and FMO conceptualized and developed the manuscript. MF, AMA, DN, and IA reviewed the manuscript.

\section{References}

1. Hotonu SA, Johnson CHN, Kansal N, Bhattacharya V (2018) Common femoral artery aneurysm repair using bifurcated graft. BMJ Case Rep 2018: bcr2017223331.

2. Koch AE, Rigdon EE (1992) Aneurysms of the superficial femoral artery: A report of two cases and review of the literature. J Vasc Surg 16: 790-793.

3. Piffaretti G, Mariscalco G, Tozzi M, Rivolta N, Annoni M, et al. (2011) Twenty-year experience of femoral artery aneurysms. J Vasc Surg 53: 1230-1236.

4. Hirsch AT, Haskal ZJ, Hertzer NR, Bakal CW, Creager MA, et al. (2006) ACC/AHA 2005 practice guidelines for the management of patients with peripheral arterial disease (Lower Extremity, Renal, Mesenteric, and Abdominal Aortic). Circulation 113: e463-e654.

5. Hirsch AT, Haskal ZJ, Hertzer NR, Bakal CW, Creager MA, et al. (2006) ACC/AHA 2005 Guidelines for the Management of Patients With Peripheral Arterial Disease (Lower Extremity, Renal, Mesenteric, and Abdominal Aortic): Executive Summary A Collaborative Report From the American Association for Vascular Surgery/Society for Vas. Journal of the American College of Cardiology 47: 1239-1312.

6. Cutler BS, Darling RC (1973) Surgical management of arteriosclerotic femoral aneurysms. Surgery 74: 764-773.

7. Amer N, Grocott E, Shami S (2004) Time for a new classification of femoral artery aneurysm? VASA 33: 170172.

8. Sapienza P, Mingoli A, Feldhaus RJ, di Marzo L, Cavallari $\mathrm{N}$, et al. (1996) Femoral artery aneurysms: Long-term follow-up and results of surgical treatment. Cardiovasc Surg 4: 181-184.

9. Bandyk DF (2008) Vascular surgical site infection: Risk factors and preventive measures. Semin Vasc Surg 21: 119-123.

10. Saleem T, Baril DT (2020) Femoral aneurysm repair. StatPearls.

11. Abdel Wahab MA, Farouk N, Saleh OI (2019) Early outcomes of traumatic femoral artery aneurysm (Open Repair versus Endovascular Treatment). Ann Vasc Surg 54: $146-151$

12. Diaz A, Neal WP, Nucci AT, Ludmer P, Bitterman J, et al. (2004) Legal and ethical issues facing adolescent health care professionals. Mt Sinai J Med 71: 181-185.

13. Mohan IV, Stephen MS (2013) Peripheral arterial aneurysms: Open or endovascular surgery? Prog Cardiovasc Dis 56: 36-56.

14. Kasirajan K, Marek JM, Langsfeld M (2001) Behçet's disease: Endovascular management of a ruptured peripheral arterial aneurysm. J Vasc Surg 34: 1127-1129.

15. Lyazidi Y, Abissegue Y, Chtata H, Taberkant M (2016) Endovascular treatment of 2 true degenerative aneurysms of superficial femoral arteries. Ann Vasc Surg 30: 307.e1307.e5.

16. Mufty H, Daenens K, Houthoofd S, Fourneau I (2018) Endovascular treatment of isolated degenerative superficial femoral artery aneurysm. Ann Vasc Surg 49: 311.e11-311. e14.

17. Kimmel RM, Murphy RX Jr, Chowdary RP (1994) Optimal management of inguinal vascular graft infections. Ann Plast Surg 32: 623-629.

18. Meland NB, Arnold PG, Pairolero PC, Lovich SF (1994) Muscle-flap coverage for infected peripheral vascular prostheses. Plast Reconstr Surg 93: 1005-1011.

19. Citak MS, Cue JI, Peyton JC, Malangoni MA (1992) The critical relation- ship of antibiotic dose and bacterial 
contamination in experimental infection. J Surg Res 52 127-130.

20. Zeltsman D, Tzarnas CD, Kerstein MD (1999) Management of vascular prosthetic infections: Results of long-term follow-up. Am Surg 65: 331-333.

21. Seeger JM (2000) Management of patients with prosthetic vascular graft infection. Am Surg 66: 166-177.

22. Yeager RA, Porter JM (1992) Arterial and prosthetic graft infection. Ann Vasc Surg 6: 485-491.

23. Sumpio BE, Allie DE, Horvath KA, Marston WA, Meites HL, et al. (2008) Role of negative pressure wound therapy in treating peripheral vascular graft infections. Vascular 16: 194-200.

24. Argenta LC, Morykwas MJ (1997) Vacuum-assisted closure: A new method for wound control and treatment: Clinical experience. Ann Plast Surg 38: 563-576.

25. Adamkova M, Tymonova J, Zamecnikova I, Kadlcik M, Klosova H (2005) First experience with the use of vacuum assisted closure in the treatment of skin defects at the burn center. Acta Chir Plast 47: 24-27.
26. Saxena V, Hwang CW, Huang S, Eichbaum Q, Ingber D, et al. (2004) Vacuum-assisted closure: Microdeformations of wounds and cell proliferation. Plast Reconstr Surg 114: 1086-1096.

27. Leon LR, Taylor Z, Psalms SB, Mills JL (2008) Degenerative aneurysms of the superficial femoral artery. Eur $\mathrm{J}$ Vasc Endovasc Surg 35: 332-340.

28. Atallah C, Al Hassan HK, Neglén P (1995) Superficial femoral artery aneurysm - an uncommon site of aneurysm formation. European Journal of Vascular and Endovascular Surgery 10: 502-504.

29. Levi N, Schroeder TV (1997) Arteriosclerotic femoral artery aneurysms. A short review. J Cardiovasc Surg 38: 335-338.

30. Sharma S, Nalachandran S (2009) Isolated common femoral artery aneurysm: A case report. Cases J 2: 7522.

31. Rancic Z, Pecoraro F, Pfammatter T, Mayer D, Veith FJ, et al. (2013) Less invasive (common) femoral artery aneurysm repair using endografts and limited dissection. Eur J Vasc Endovasc Surg 45: 481-487. 